\title{
But Mouse, you are not alone: On some severe acute respiratory syndrome coronavirus 2 variants infecting mice
}

Michael J. Kuiper, PhD, is a Biomolecular Modeller who leads the Modelling and Simulations Team in at the CSIRO Data61, Dockands, Victoria, Australia

Laurence OW Wilson, PhD, is a Research Scientist who leads the Digital Genome Engineering Team at the CSIRO Transformational Bioinformatics Group, North Ryde, New South Wales, Australia

Shruthi Mangalaganesh, is studying Bachelor of Biomedical Science and Bachelor of Commerce at Monash University, Clayton, Victoria, Australia, and pursued an internship at the CSIRO Australian Centre for Disease Preparedness, Geelong, Victoria, Australia

Carol Lee, BAnVetBioSci(Hons), PhD, is CERC Postdoctoral Research Fellow in the CSIRO Transformational Bioinformatics Group, North Ryde, New South Wales, Australia

Daniel Reti, BE(Hons), BS, worked as a Research Technician at the CSIRO Transformational Bioinformatics Group, North Ryde, New South Wales, Australia, and is currently Front-End Software Engineer at the Centre for Population Genomics, joint initiative of the Garvan Institute of Medical Research, Sydney, New South Wales, Australia and the Murdoch Children's Research Institute, Melbourne, Victoria, Australia

Seshadri S Vasan, DPhil, is a Senior Principal Research Consultant and COVID-19 Project Leader at the CSIRO Australian Centre for Disease Preparedness, Geelong, Victoria, Australia, and Honorary Professor in the Department of Health Sciences at the University of York, York, UK

Address correspondence to Professor Seshadri S Vasan, CSIRO Australian Centre for Disease Preparedness, 5 Portarlington Road, Geelong, 3220, Victoria, Australia or email vasan.vasan@csiro.au

NB: MJK and LOWW contributed equally to this paper.

Running Title: On some SARS-CoV-2 variants infecting mice

\section{Abstract}

In silico predictions combined with in vitro, in vivo and in situ observations collectively suggest that mouse adaptation of the SARS-CoV-2 virus requires an aromatic substitution in position 501 or position 498 (but not both) of the spike protein's receptor binding domain. This effect could be enhanced by mutations in positions 417, 484, and 493 (especially K417N, E484K, Q493K and Q493R), and to a lesser extent by mutations in positions 486 and 499 (such as F486L and P499T). Such enhancements due to more favourable binding interactions with residues on the complementary angiotensin-converting enzyme 2 (ACE2) interface, are however, unlikely to sustain mouse infectivity on their own based on 
theoretical and experimental evidence to date. Our current understanding thus points to the Alpha,

37 Beta, Gamma, and Omicron variants of concern infecting mice, while Delta and 'Delta Plus' lack a similar

38 biomolecular basis to do so. This paper identifies eleven countries (Brazil, Chile, Djibouti, Haiti, Malawi,

39 Mozambique, Reunion, Suriname, Trinidad and Tobago, Uruguay and Venezuela) where targeted local

40 field surveillance of mice is encouraged because they may have come in contact with humans who had

41 the virus with adaptive mutation(s). It also provides a systematic methodology to analyze the potential

42 for other animal reservoirs and their likely locations.

\section{Keywords}

AlphaFold; Animal reservoir; COVID-19; in silico; in vitro; in vivo; mouse adaptation; SARS-CoV-2; variants

\section{Introduction}

48 The 'novel coronavirus disease' (COVID-19) has resulted in significant global morbidity and mortality on

49 a scale similar to the influenza pandemic of $1918 .{ }^{1}$ The ongoing pandemic has been sustained through

50 the activities of human beings, who are the largest reservoir of the causative 'severe acute respiratory

51 syndrome virus 2' (SARS-CoV-2). This RNA virus in a new host (human beings) is evolving rapidly,

52 accumulating mutations, and existing as a cloud of variants with quasispecies diversity. ${ }^{2}$ Last year, the

53 world witnessed the risk of this virus acquiring additional reservoirs (such as minks) and new mutations

54 of consequence (such as 'Cluster 5'), which could increase transmissibility and lead to a potentially

55 weaker antibody response. ${ }^{3}$

57 Both the original form of the virus (known as 'D614') and the subsequent more transmissible 'G614'

58 variant which has replaced it almost entirely in circulation, ${ }^{4,5}$ did not infect mice because their

59 'angiotensin-converting enzyme 2' (ACE2) receptor did not bind the viral spike protein effectively to 
60 allow entry into cells. Since mouse (Mus musculus) is a popular animal model of infection, the virus had

61 to be adapted through techniques such as sequential passaging in mouse lung tissues and modifying the

62 receptor binding domain $(\mathrm{RBD}) \cdot{ }^{6-8}$ Other strategies to infecting mice with the original form of the virus or

63 the G614 included transgenic mice expressing human ACE2 (hACE2), and sensitizing the mouse

64 respiratory tract through transduction with adenovirus or adeno-associated virus expressing hACE2. ${ }^{9-12}$

65 Recently, virus 'variants of concern' (VOC) originating from Brazil, South Africa and the UK (which

66 contain the common mutation N501Y in the RBD) were shown to infect mice. ${ }^{13,14}$ This ability of SARS-

67 CoV-2 variants of concern to infect mice is unsettling because of the potential to establish additional

68 reservoirs in a species that is in close contact with people and companion animals, especially as its

69 population is hard to vaccinate or control.

\section{Methodology}

72 In this paper, we have combined our structural predictions from biomolecular modelling with available

73 experimental evidence to understand how specific mutations in VOC and mouse adapted strains,

74 especially in the RBD, have enabled this virus to infect mice. To do this, we compared the spatial

75 interactions of these key mutations with the corresponding regions in both mouse ACE2 (mACE2) and

76 hACE2 using homology molecular models based on cryogenic electron microscopy (cryo-EM) data of the

77 ACE2/RBD interface (protein database 'pdb' entry number $6 \mathrm{M} 17) .{ }^{15}$ We have assumed the coordinates

78 of ACE2 and RBD in the 6M17 structure to be reasonable starting positions for our respective models,

79 with root-mean-square deviation (RMSD) scripts alignment based on C-Alpha carbon protein backbones.

80 As the protein prediction software AlphaFold has been recently been released, ${ }^{16}$ we also recalculated

81 RBD structures, finding Alphafold predictions to be in excellent agreement with our initial homology

82 models, with less than $0.9 \AA$ RMSD average alignment. Amino acid side chain conformations of the RBD

83 models were adapted from AlphaFold predictions as they were deemed more reliable. 
85 By introducing the ACE2 models and variants into molecular dynamics simulations to optimize the

86 interactions, similar to our recent work, ${ }^{17,18}$ we qualitatively identified the key interactions of side chain

87 residues at the ACE2/RBD interface. Further details are provided under 'Supplementary Methods for

88 Molecular Modelling'. We then compared our in-silico findings with experimental results reported by

89 different research groups with mouse adapted strains/isolates ${ }^{6-8,13,21-29}$ and VOC ${ }^{14,30-34}$ (Tables 1 and 2).

90 Taken together, we were able to gain valuable insights into the likely effects of different mutations of

91 consequence.

93 Subsequently, we queried the world's largest public database called 'GISAID' (Global Initiative on

94 Sharing All Influenza Data) and looked for these mutations, and equivalent mutations of consequence.

95 This 'Big Data', comprising of circa 2.4 million and 4 million SARS-CoV-2 genome sequences as of 21 July

96 and 4 October 2021 respectively, formed the basis of our in situ analysis. ${ }^{29}$ Over $99.9 \%$ of these

97 sequences are from viruses sequenced from human hosts. In brief, sequences were aligned to the back

98 to the SARS-CoV-2 reference (EPI_ISL_402124, denoted as the WT allele) in order to generate a file

99 containing all mutations in the variant call format (VCF), which is a concise way for storing gene

100 sequence variations. We then calculated the ratio of the frequency at which the WT allele versus the

101 mutant allele were observed across a rolling 14-day window. Given the data is discrete, highly variable in

102 size across countries, and contains background noise, such a window of time is essential from our

103 experience to reduce distortions and glean meaningful insights. For example, if 14 WT and 28 mutant

104 sequences were observed within a specified 14-day window, then the frequencies were 1/day and 2/day

105 respectively. The ratio of mutant:WT frequency is therefore 2, indicating the mutant allele is appearing

106 twice as frequently as the WT allele within that period. This was used to create a heatmap for the key

107 mutations, both individually and in combination (as discussed below). For clarity, we only considered 
countries where the mutant:WT ratio exceeded 1.2 across the entire time the mutant was sampled or

109 within any given 14-day window. To reduce the noise further from low-sampled countries, we also

110 instituted a minimum threshold of $10 \mathrm{WT}$ and 10 mutant samples over at least 14 days to suggest

111 possible spread locally. For this reason, a country where a mutant had only been recorded on a single

112 day, or where only 9 mutant samples were recorded overall, was not included in our analysis. Our

113 heatmap scale has also been truncated at 2.00 for ease of visual comparison.

115 Results and Discussion

\section{In silico results}

117 In silico comparison of the interface residues of the RBD/ACE2 complex in human and mouse models

118 reveals 30 ACE2 residues in close contact, of which 19 are conserved between mACE2 and hACE2

119 (approximately 63\% identity for the contacting ACE2 residues). The RBD mutations associated with the 120 mouse adaptations listed in Table 1 can be grouped loosely into 3 regions by their positions on the 121 ACE2/RBD interface as follows: Region 1 (RBD positions 498, 499 and 501) are centered around the 122 highly conserved ACE2 residue tyrosine 41 (Y41); Region 2 (RBD positions 417, 493) centered around 123 ACE2 residue 34; and Region 3 (RBD positions 484 and 486) close to a cluster of ACE2 residues 78 to 82.

124 ACE2 residues within $5 \AA$ (chosen to account for molecular fluctuations) of each RBD adaptation are 125 listed in Table 3, with dissimilar human/mouse ACE2 residues highlighted in yellow. Figure 1 further 126 illustrates these residues visually, and how they are relatively positioned in three regions. Figure 2 aligns 127 the human and mouse ACE2 to highlight the key differences at the contact points with the RDB (shown 128 in yellow).

130 Modelling the N501Y mutation at the ACE2/RBD interface reveals a close interaction with the highly 131 conserved Y41 residue in ACE2, through attractive, non-covalent bonding between aromatic amino acids 
132 known as $\pi$-stacking interactions. In mACE2, $\pi$-stacking can be enhanced by the proximal substitution of

133 histidine $(\mathrm{H})$ in place of lysine $(\mathrm{K})$ which is present at position 353 of hACE2. Our modelling also shows

134 similar $\pi$-stacking enhancement with the conserved $Y 41$, through the substitution of RBD glutamine at

135 position $498(\mathrm{Q} 498)$ to either histidine $(\mathrm{H})$ or tyrosine $(\mathrm{Y})$. These aromatic $\pi$-stacking interactions appear

136 to be reasonably strong as $\mathrm{N} 501 \mathrm{Y}$ and $\mathrm{Q} 498 \mathrm{H}$ can each sustain mouse adaptation on its own in

137 experiments. ${ }^{6,13,23,28}$ Counterintuitively, our modelling predicts that simultaneous aromatic mutations at

138 RBD positions 498 and 501 is detrimental to mouse adaptation due to local $\pi$-stacking distortion to the

139 binding interface. This could explain why very few simultaneous aromatic mutations at these positions

140 have been observed. In over 2.4 million entries on GISAID as of 21 July 2021, we detected only a single

141 co-occurrence, that of an adapted isolate from mouse lung homogenate where N501Y occurred with

142 glutamate 498 to arginine (Q498R). ${ }^{29}$ However, when we recently re-run our in situ model with over 4

143 million human-origin GISAID entries, we detected 0, 2 and 14 instances of Y501 co-existing with Y498,

$144 \mathrm{H} 498$ and R498 respectively. The two instances of Y501-H498 are low quality and/or low coverage

145 sequences; the 14 instances of Y501-R498 have been reported from France, Netherlands, South Africa,

146 Spain, UK and USA between March and September 2021. Although arginine is not aromatic, it is

147 frequently associated with $\pi$-stacking interactions with inherent conformational flexibility compared to

148 tyrosine or histidine, therefore it is likely that the Y501-R498 combination is more tolerated (for

149 example, with the recent Omicron variant of concern). ${ }^{37}$

150

151 In Region 1 (Table 3) we also note the proline 499 to threonine (P499T) substitution in two infectious

152 clones, presumably engineered to enhance Y498. Modelling provides the following insight on this: the

153 change from $\mathrm{P}$ to $\mathrm{T}$ will relax the backbone constraints of proline and allow conformational

154 rearrangement of threonine to contact the conserved ACE2 residues Y41 and L45. However, we don't

155 find any strong molecular modelling basis for this mutation to sustain mouse adaptation on its own or 
evolve naturally alongside adaptive mutations at positions 498 or 501; this is borne out by experimental

157 evidence to date. In other words, in silico predictions combined with in vitro and in vivo evidence

158 collectively suggest that mouse adaptation requires an aromatic substitution in either position 501 or

159 position 498 (but not both); while additional mutations, especially in Region 2 and Region 3 of the RBD

160 as summarized below, enhancing ACE2 binding interactions and specificity in mice. These predictions

161 are further supported by AlphaFold that assigns very high confidence scores (>93 in a scale of 0-100) for

162 the structural predictions involving these key mutations of the individual proteins, however predictions

163 of the RBD/ACE2 complex are currently sub-optimal. With time, the quality of AlphaFold predictions of

164 protein complexes will improve thanks to additional experimental observations, and thus it is expected

165 to play a more central role in structural interpretation during pandemics such as COVID-19 and future

166 'Disease-X'.

From Table 1, we see that mouse adapted strains sometimes carry the Q493K/R mutation (polar

171 conserved in hACE2 as well as mACE2 (c.f. Table 3, Region 2). The K417N substitution (lysine to

172 asparagine), which is another experimental observation from Table 1, is also predicted by modelling to

173 be advantageous for mouse adaptation due to favourable amide hydrogen bond interactions with

174 interfacial mACE2 residues asparagine 30 (N30) and glutamine 34 (Q34); such amide hydrogen bonding

175 is not possible in hACE2 as it has non-amide lysine $(\mathrm{K})$ and histidine $(\mathrm{H})$ residues, as also noted by other

176 researchers. ${ }^{21}$ With the Gamma variant of concern, it is unclear whether the K417T enhances the role of

177 N501Y in mouse adaptation in a similar manner. The 'Delta Plus' variant of concern has the K417N

178 mutation, but there is no molecular modelling basis to believe that it can infect mice without an

179 aromatic change in position 498 or 501 as described above. It would be worthwhile to further 
180 investigate any interfering role of glycosylation at this interface region, because hACE2 contains $\mathrm{N}$-linked

181 glycosylation at asparagine 90 (N90) whereas mACE2 does not (its analogous residue is threonine T90

182 according to Uniprot references Q8R0I0 and Q9BYF1).

184 In Region 3, the K484 residue is not positioned directly at the interface and not observed to interact

185 strongly with any ACE2 residues; however, our model shows occasional salt bridges can be formed with

186 relatively close glutamic acid residues in positions 35 and 75 that are conserved in both hACE2 and

187 mACE2. Our simulations show that the distance between $\mathrm{K} 484$ and these glutamic acid residues

188 fluctuate dynamically from 3 to $20 \AA$, with salt bridges more likely when distances are around $3 \AA$. Thus,

189 the E484K, which is present in the Beta and Gamma variants of concern, and more recently in some

190 Alpha isolates as well, is likely to have an enhancing role through transient salt bridges. The same cannot

191 be said about E484Q seen in the Delta variant of concern because salt bridge formation is unlikely with

192 the polar glutamine (Q) residue. With no accompanying aromatic change in positions 498 or 501 , we

193 believe that the $\mathrm{E} 484 \mathrm{Q}$ in Delta, and additionally the $\mathrm{K} 417 \mathrm{~N}$ in 'Delta Plus', cannot sustain mouse

194 infectivity on their own based on current biomolecular understanding. It also follows that the Omicron

195 variant of concern is expected to infect wild type mice because it has the essential and enhancing

196 mutations. The residues 75 to 82 in mACE2 are significantly different from hACE2 (Table 3), therefore

197 any mutation in the corresponding RBD interface is worth investigation. We could find one from

198 experimental observations, the engineered substitution $\mathrm{F} 486 \mathrm{~L},{ }^{27}$ and consider it to have at best an

199 enhancing role. As it was observed simultaneously with Q498Y (which is likely to sustain mouse

200 infectivity on its own), the contribution of F486L to the overall mouse adaptation remains to be

201 ascertained.

202

203 Comparison with in vitro, in vivo and in situ observations 
Early in silico predictions based on comparative structural analysis of ACE2 suggested that mouse has a

very low probability of being infected. ${ }^{35,36}$ Although correct about mouse, those analyses also made inconsistent and erroneous predictions that ferrets wouldn't be susceptible, pigs would be susceptible,

207 etc., thus exposing the need for experimental inputs into the model. Therefore, this paper takes into

208 account a range of experimental observations to cross-check our in silico predictions through

209 biomolecular modelling. Wan et al. reasoned that "mouse or rat ACE2 contains a histidine at the 353

210 position which does not fit into the virus-receptor interaction as well as a lysine does". ${ }^{35}$ While this is

211 true of the original Wuhan strain containing asparagine 501 (N501) in the RBD, our modelling indicates

212 why the tyrosine 501 mutation enables mouse infectivity, even on its own. In hACE2, lysine 353 (K353)

213 creates a salt bridge with conserved aspartic acid 38 (D38). In mACE2, lysine 353 is replaced by the

214 aromatic histidine ( $\mathrm{H} 353)$ to complete the salt bridge, as well as contribute to $\pi$-stacking with Y501

215 variant. The N501Y mutation will also lead to favorable $\pi$-stacking with the highly conserved tyrosine 41

216 (Y41) residue in mammalian ACE2, as suggested by Starr et al. ${ }^{38}$ with deep scanning of RBD mutations

217 and hACE2 affinity assays. These authors highlighted enhanced affinity of F501 (as it had the highest

218 score), followed by Y501, V501, W501 and T501, in that order. But Y501 and T501 require only a single

219 nucleotide change and have been observed more frequently in situ (Figure S1a) - compared to F501,

220 V501 and W501 which require 2, 2 and 3 nucleotide changes respectively. It is unsurprising that the

221 latter variants requiring two or more changes were rarely observed in situ regardless of their high in

222 vitro affinity scores from Starr et al. ${ }^{38}$

224 From the above and Table 4, we see that in silico analysis can provide valuable insights to interpret and

225 bridge in vitro, in vivo and in situ observations on the RBD position 501. A similar analysis is possible with

226 the alternative essential mutation for mouse adaptation at RBD position 498, where the in vitro affinity

227 enhancement order is $\mathrm{H} 498, \mathrm{Y} 498, \mathrm{~F} 498$ and W498 according to Starr et al. ${ }^{38}$ Of these, $\mathrm{H} 498$ (on its own) 
and Y498 (with enhancing RBD mutations) have been shown to result in mouse adaptation in vivo (Table 1) ${ }^{7,8,23-28} \mathrm{Q} 498 \mathrm{R}$ was also reported once, unusually in combination with $\mathrm{N} 501 \mathrm{Y}$, isolated from mouse lung after 30 passages. In humans, in situ observations of these variants have been limited to R498 (57 occurrences) and H498 (8 occurrences) so far. Thus, it is clear from in vitro, in vivo and in situ analyses (Table 4) that H498, R498 and Y498 are possible but not yet common. This is consistent with our in silico predictions because H498 and Y498 are aromatic (enabling $\pi$-stacking with ACE2 Y41; similar to Y501),

234 while R498 has conformational flexibility and can still be associated with $\pi$-stacking interactions. ${ }^{37} \mathrm{H} 498$ 235 and R498 observed in situ require a single nucleotide change from Q498, while Y498 requires two 236 nucleotide changes (or one change from $\mathrm{H} 498$ ). and in situ observations, we see that K417M is less common than K417N or K417T (Figures S2a and S2b),

240 although in vitro studies did not predict any enhancement. ${ }^{38}$ In silico predictions show that all these 241 require a single nucleotide change, but that N417 (and Q417) would benefit from amide hydrogen 242 bonding. E484K is present in Beta and Gamma (Figure S2c), and increasingly in Alpha VOC, while E484Q 243 is present in the Kappa variant of interest that is related to the Delta VOC. In comparison to these two 244 substitutions and notwithstanding higher in vitro affinity scores, R484 and T484 are infrequently 245 observed in situ, which is consistent with our in silico predictions because they each require 2 nucleotide 246 changes from E484, or one change from K484. The F486L and P499T were originally engineered in vivo,

247 but have had sporadic in situ presence in human populations. In silico predictions suggest that the F486L 248 mutation (accessible by three possible ways of a single nucleotide change) can aid mACE2 adaptation, 249 due to the human-mouse differences in ACE2 at the 78-82 region; the P499T is also a single nucleotide 250 change (but only one way from $\mathrm{P}$ to $\mathrm{T}$ ) and predicted to be rare in comparison. Finally, in silico 251 predictions for Q493 substituted by K, L or R (each a single nucleotide change) are borne out in vivo and 
in situ, although their affinity scores from in vitro experiments are low. The affinity scores from Starr et

$253 a .^{38}$ were developed for hACE2 (not mACE2), so we expected a greater correlation than what has been

254 observed in situ in human populations, but perhaps it is still early in the pandemic to assess this

255 definitively. It also looks likely that there are factors other than enhancement of ACE2 binding that

256 determine susceptibility - as seen from Starr et $a .^{38}$ and Table 4; from our own inconclusive attempts at

257 correlating free energy binding affinities using in silico methods (not shown); and from a more detailed

258 in silico model by Piplani et al..$^{39}$ which counter-intuitively predicts lower binding free energy for dogs

259 compared to more susceptible animals such as monkeys, hamsters, ferrets, cats and tigers. ${ }^{40}$

261 Some mutations in the essential as well as enhancing positions can lead to other mutations. For

262 example, N501Y, the key mutation common to the Alpha, Beta, and Gamma variants of concern, can

263 lead to F501 with a further nucleotide change. Similarly, the enhancing E484K mutation can also lead to

264 R484 or T484 with a further nucleotide change. We examined whether in situ observations are

265 consistent or contrary to our in silico predictions. Indeed, F501 was observed in Sweden (28 April 2021),

266 Germany (7 May 2021), Mexico (22 June 2021) and Colombia (30 June 2021), once in each of these four

267 countries, while the Y501 has been observed in these countries since 12 March 2020, 21 October 2020,

26831 January 2021 and 19 September 2020 respectively. UK reported E484R in August 2020, followed by

269 Angola in April 2021; Brazil and USA in May 2021; South Korea in June 2021; and South Africa in July

270 2021. In each case, the E484K was detected prior to E484R - the former mutation circulating in UK,

271 Angola, Brazil, USA, South Korea and South Africa from April, August, April, March, December and

272 August 2020 respectively. Similarly, E484T was only recently detected in the USA in June 2021, 15

273 months after the first report of E484K in that country. All eleven instances are thus consistent with our

274 prediction - whether this link is causal or a coincidence is worthy of investigation with local

275 epidemiological data. While bioinformatics tools can provide useful insights, out of 53 COVID-positive 
cases only one sample is on average sent for virus genome sequencing (as of 4 October 2021), with huge variations across time and locations, and lots of missing meta-data. ${ }^{41,42}$ This means we are more confident about ruling in (e.g. when a variant has been detected in a location) than ruling out the possibility of a mutation circulating purely based on in silico data, even if the latter is statistically large (4 million as of 4 October 2021).

Our analysis is not just of theoretical interest; it has huge practical applications because mice can be

283 kept as pets, or come into contact with other pets like cats which are known to be susceptible. Also,

284 mouse plague can occur in area of COVID-19 outbreaks or endemicity, as is currently the case in New

285 South Wales and adjacent states of Australia. ${ }^{43}$ In order to help public health and animal health

286 professionals, Figures $3 a$ and $3 b$ show the eleven countries where key essential and enhancing

287 mutations listed in Tables 1 and 4 (viz. N501Y, E484K and K417N/T) have co-occurred. The underlying

288 raw data, down to regional counts for these combinations, are available from

289 https://www.biorxiv.org/content/10.1101/2021.08.04.455042v2.supplementary-material. We believe

290 that this information will help locate areas at risk (especially in Brazil, Chile, Djibouti, Haiti, Malawi,

291 Mozambique, Reunion, Suriname, Trinidad and Tobago, Uruguay and Venezuela) for appropriate

292 mitigation measures.

294 Conclusion and further analyses

295 Assessing the risk of viruses adapting to new hosts requires careful interpretation of all available data

296 from in silico, in vivo, in vitro and in situ sources. Understanding host adaptation at a molecular level, via 297 modelling helps reconcile seemingly conflicting, experimental, and clinical observations while a 298 pandemic is still in progress. We have demonstrated this with the SARS-CoV-2 virus adapting to mice.

299 Our conclusions come with humility, as they are based on best available evidence up to this point, but 
allowing us and others to refine when more evidence becomes available. Armed with our collective understanding from different approaches, and bolstered by bioinformatics and emerging artificial intelligence technologies such as Alphafold, we have shown how to position ourselves better to predict and mitigate virus host adaptations, not just for this pandemic but also for future Disease-X. Further analyses pertaining to COVID-19 should focus on the role of mutations beyond the spike and RBD; experimentally prove that the Omicron variant of concern can infect wild type mice; improve computational modelling of binding affinities to explore correlations with susceptibility, if any; assess the actual risk of transmission for this virus through aerosol versus other routes; and study other hosts

308 like rats and other potential reservoir species (even those that previously exhibited low receptor activities) which will be hard to vaccinate or control..$^{35,36,44}$

\section{Supplementary Methods for Molecular Modelling}

312 Molecular simulations were performed using NAMD2.14 $4^{45}$ with CHARM $36 m^{46}$ forcefield employing a

313 'TIP3' water model. The SARS-CoV-2 spike/ACE2 model was a homology model based on one of the best

314 pdb structures available at the time of our analysis, viz. 6M17, which is deemed to be of sufficient

315 quality for our purpose. ${ }^{15}$ Variant models of the SARS-CoV-2 spike RBD domain (residues 330 to 530)

316 containing the mouse adapted mutations were constructed by mutating residues in the NAMD build

317 scripts, but later found to be in very close agreement with the same model constructed using

318 AlphaFold ${ }^{16}$ (less than $0.9 \AA$ RMSD difference to C-Alpha backbone atoms). A truncated mACE2

319 consisting of residues 19 to 600 was built using Swiss modeller, ${ }^{47}$ and similarly found to be in close

320 agreement with the equivalent AlphaFold model. Amino acid side chain conformations predicted by

321 Alphafold were used in all initial RBD model starting conformations. Glycosylation of the spike and

322 mACE2 protein was manually constructed using Visual Molecular Dynamics (VMD). Simulations were

323 run with Periodic Boundary Conditions 'PBCs' using the 'NPT' isothermal-isobaric ensemble at 310K and 
3241 bar pressure employing Langevin dynamics. The PBCs were constant in the XY dimensions. Long-range

325 Coulomb forces were computed with the Particle Mesh Ewald method with a grid spacing of $1 \AA$. 2 fs

326 timesteps were used with non-bonded interactions calculated every 2 fs and full electrostatics every $4 \mathrm{fs}$

327 while hydrogens were constrained with the 'SHAKE' algorithm. The cut-off distance was $12 \AA$ with a

328 switching distance of $10 \AA$ and a pair-list distance of $14 \AA$. Pressure was controlled to 1 atmosphere

329 using the Nosé-Hoover Langevin piston method employing a piston period of $100 \mathrm{fs}$ and a piston decay

330 of 50 fs. Trajectory frames were captured every 100 ps. Eleven variant models were constructed

331 representing the mouse-adapted variants observed in Table $\mathbf{1}$ as well as the original Wuhan RBD model

332 with both mouse and human ACE2. Models were simulated for 300 nanoseconds. Trajectories were

333 visualized with VMD and Nanome. Modelling data shall be made available on the CSIRO Data access

334 portal (https://data.csiro.au/).

336 Acknowledgements

337 This work was supported by funding (Principal Investigator: S.S.V.) from the Australia's Department of

338 Finance, CSIRO Future Science Platforms, National Health and Medical Research Council (MRF2009092),

339 and United States Food and Drug Administration (FDA) Medical Countermeasures Initiative contract

340 (75F40121C00144). The article reflects the views of the authors and does not represent the views or

341 policies of the funding agencies including the FDA. We are grateful for support from our colleagues at

342 the Australian Centre for Disease Preparedness (https://www.grid.ac/institutes/grid.413322.5)

343 (especially Simran Chahal, Trevor Drew, Alexander McAuley and Nagendrakumar Singanallur) and the

344 Transformational Bioinformatics Group (especially Denis Bauer, Yatish Jain, Brendan Hosking and Aidan

345 Tay). L.O.W.W acknowledges grant funding from the Australian Academy of Science and Australia's

346 Department of Industry, Science, Energy and Resources. The title is from the poem 'To a Mouse: On

347 Turning her up in her Nest, with the Plough, November $1785^{\prime}$ by Scotland's national poet Robert Burns, 
348 in which he says that the mouse is not alone in proving foresight may be vain as the best-laid schemes of

349 mice and men go oft awry (But, Mousie, thou art no thy-lane, In proving foresight may be vain: The best-

350 laid schemes o' Mice an' Men Gang aft agley).

351

352 Author contributions

353 Conceptualization, methodology, and funding acquisition, S.S.V.; in silico analysis, M.J.K.; in vitro

354 analysis, M.J.K. and S.S.V.; in vivo analysis, S.M. and S.S.V.; in situ analysis, L.O.W.W., D.R. and C.L.;

355 writing - original draft preparation, S.S.V, M.J.K. and S.M.; writing - review and editing, all authors.

356

357 References

358 1. Dong E, Du H, Gardern L. An interactive web-based dashboard to track COVID-19 in real time.

359 Lancet Infectious Diseases. 2020;20(5):533-534. Available at https://doi.org/10.1016/S1473-

360 3099(20)30120-1 (accessed 1 December 2020).

361 2. Bauer DC, Tay AP, Wilson, LOW, et al. Supporting pandemic response using genomics and

362 bioinformatics: A case study on the emergent SARS-CoV-2 outbreak. Transboundary \& Emerging

363 Diseases. 2020; 67(4):1453-1462. Available at https://doi.org/10.1111/tbed.13588 (accessed 1

364 December 2020).

$3653 . \quad$ Lassaunière R, Fonager J, Rasmussen, $M$, et al. Working paper on SARS-CoV-2 spike mutations

366 arising in Danish mink, their spread to humans and neutralization data. 2020. Available at

367 https://files.ssi.dk/Mink-cluster-5-short-report_AFO2 (accessed 1 December 2020).

368 4. Korber B, Fischer WM, Gnanakaran S, et al. Tracking changes in SARS-CoV-2 spike: Evidence that

369 D614G increases infectivity of the COVID-19 virus. Cell. 2020; 182(4):812-827. Available at

370 https://doi.org/10.1016/i.cell.2020.06.043 (accessed 1 December 2020). 
371 5. Zhang J, Cai Y, Xiao T, et al. Structural impact on SARS-CoV-2 spike protein by D614G

372 substitution. Science. 2021; 372(6541):525-530. Available at

373 https://doi.org/10.1126/science.abf2303 (accessed 1 May 2021).

3746 Gu H, Chen Q, Yang G, et al. Adaptation of SARS-CoV-2 in BALB/c mice for testing vaccine

375 efficacy. Science. 2020; 369(6511):1603-1607. Available at

$376 \quad$ https://doi.org/10.1126/science.abc4730 and

377 https://www.biorxiv.org/content/10.1101/2020.05.02.073411v1 (accessed 1 December 2020).

378 7. Dinnon KH, Leist SR, Schäfer A, et al. A mouse-adapted model of SARS-CoV-2 to test COVID-19

379 countermeasures. Nature. 2020; 586:560-566. Available at https://doi.org/10.1038/s41586-020-

380 2708-8 (accessed 1 December 2020).

381 8. Leist SR, Dinnon KH, Schäfer A, et al. A mouse-adapted SARS-CoV-2 induces acute lung injury

382 and mortality in standard laboratory mice. Cell. 2020; 183:1070-1085. Available at

383 https://doi.org/10.1016/i.cell.2020.09.050 (accessed 1 December 2020).

384 9. Yuan L, Tang Q, Cheng T, Xia N. Animal models for emerging coronavirus: progress and new

385 insights. Emerging Microbes \& Infections. 2020; 9(1):949-961. Available at

$386 \quad$ https://doi.org/10.1080/22221751.2020.1764871 (accessed 15 May 2021).

387 10. Muñoz-Fontela C, Dowling WE, Funnell SGP, et al. Animal models for COVID-19. Nature. 2020;

388 586: 509-515. Available at https://doi.org/10.1038/s41586-020-2787-6 (accessed 1 December

$3892020)$.

390 11. Zeiss CJ, Compton S, Veenhuis, RT. Animal models of COVID-19. I. Comparative virology and

391 disease pathogenesis. ILAR Journal. 2021; ilab007. Available at

$392 \quad$ https://doi.org/10.1093/ilar/ilab007 (accessed 1 May 2021).

393 12. Veenhuis RT, Zeiss CJ. Animal models of COVID-19 II. Comparative immunology. ILAR Journal.

394 2021; ilab010. Available at https://doi.org/10.1093/ilar/ilab010 (accessed 1 May 2021). 
13. Rathnasinghe R, Jangra S, Cupic A, et al. The N501Y mutation in SARS-CoV-2 spike leads to morbidity in obese and aged mice and is neutralized by convalescent and post-vaccination human sera. medRxiv. 2021; 2021.01.19.21249592. Available at https://doi.org/10.1101/2021.01.19.21249592 (accessed 1 February 2021).

14. Yao W, Wang Y, Ma D, et al. Circulating SARS-CoV-2 variants B.1.1.7, 501Y.V2, and P.1 have gained ability to utilize rat and mouse Ace2 and altered in vitro sensitivity to neutralizing antibodies and ACE2-Ig. bioRxiv. 2021; 2021.01.27.428353. Available at https://doi.org/10.1101/2021.01.27.428353 (accessed 1 May 2021).

15. Yan R, Zhang Y, Li Y, et al. Structural basis for the recognition of SARS-CoV-2 by full-length human ACE2. Science. 2020; 367:1444-1448. Available at https://doi.org/10.1126/science.abb2762 (accessed 1 December 2020).

406 16. Jumper, J., Evans, R., Pritzel, A. et al. Highly accurate protein structure prediction with AlphaFold. Nature (2021). Available at https://doi.org/10.1038/s41586-021-03819-2 (accessed 20 July 2021).

409 17. McAuley AJ, Kuiper MJ, Durr PA, et al. Experimental and in silico evidence suggests vaccines are unlikely to be affected by D614G mutation in SARS-CoV-2 spike protein. Npj Vaccines. 2020;

412 18. Riddell S, Goldie S, McAuley A, et al. Live virus neutralisation of the 501Y.V1 and 501Y.V2 SARSCoV-2 variants following INO-4800 vaccination of ferrets. bioRxiv. 2021; 2021.04.17.440246. Available at https://doi.org/10.1101/2021.04.17.440246 (accessed 1 May 2021).

415 19. Tai W, He L, Zhang X. Characterization of the receptor-binding domain (RBD) of 2019 novel 416 coronavirus: implication for development of RBD protein as a viral attachment inhibitor and 417 vaccine. Cellular \& Molecular Immunology. 2020; 17:613-620. Available at 
419 20. Grantham, R. Amino acid difference formula to help explain protein evolution. Science. 1974;

420 185(4154): 862-864. Available at https://doi.org/10.1126/science.185.4154.862 (accessed 1

421 December 2020).

422 21. Sun S, Gu H, Cao L, et al. Characterization and structural basis of a lethal mouse-adapted SARS-

423 CoV-2. Nature Communications. 2021; 12: 5654. Available at

424 https://www.nature.com/articles/s41467-021-25903-x (accessed 30 September 2021).

425 22. Fagre A, Lewis J, Eckley M, et al. SARS-CoV-2 infection, neuropathogenesis and transmission

426 among deer mice: Implications for spillback to New World rodents. PLoS Pathogens. 2021; 17(5):

427 e1009585. Available at https://doi.org/10.1371/journal.ppat.1009585 (accessed 24 May 2021).

428 23. Wang J, Shuai L, Wang C, et al. Mouse-adapted SARS-CoV-2 replicates efficiently in the upper 429 and lower respiratory tract of BALB/C and C57BL/6J mice. Protein \& Cell. 2020; 11:776-782. $430 \quad$ Available at https://doi.org/10.1007/s13238-020-00767-x (accessed 1 December 2020).

431 24. Liu Z, Zheng H, Lin H, et al. Identification of common deletions in the spike protein of Severe

434 25. Zhou H, Chen X, Hu T, et al. A novel bat coronavirus closely related to SARS-CoV-2 contains 435 natural insertions at the S1/S2 cleavage site of the spike protein. Current Biology. 2020; 436 30:2196-2203. Available at https://doi.org/10.1016/i.cub.2020.05.023 (accessed 1 December 437 2020).

438 26. Huang K, Zhang Y, Hui X, et al. Q493K and Q498H substitutions in spike promote adaptation of 439 SARS-CoV-2 in mice. EbioMedicine. 2021; 67:103381. Available at $440 \quad$ https://doi.org/10.1016/i.ebiom.2021.103381 (accessed 24 May 2021). 
441 27. Muruato A, Vu MN, Johnson BA, et al. Mouse adapted SARS-CoV-2 protects animals from lethal

442 SARS-CoV challenge. bioRxiv. 2021; 2021.05.03.442357. Available at

$443 \quad$ https://doi.org/10.1101/2021.05.03.442357 (accessed 15 May 2021).

444 28. Zhang $\mathrm{Y}$, Huang $\mathrm{K}$, Wang $\mathrm{T}$, et al. SARS-CoV-2 rapidly adapts in aged BALB/c mice and induces

445 typical pneumonia. Journal of Virology. 2021;95(11):e02477-20. Available at

446 https://doi.org/10.1128/JVI.02477-20 (accessed 24 May 2021).

447 29. Van Noorden, R. Scientists call for fully open sharing of coronavirus genome data. Nature. 2021; 590:195-196. Available at https://doi.org/10.1038/d41586-021-00305-7 (accessed 1 March 2021).

30. Rambaut A, Loman N, Pybus O, et al. Preliminary genomic characterisation of an emergent SARS-CoV-2 lineage in the UK defined by a novel set of spike mutations. Virological. 2020. Available at https://virological.org/t/preliminary-genomic-characterisation-of-an-emergent-sarscov-2-lineage-in-the-uk-defined-by-a-novel-set-of-spike-mutations/563 (accessed 1 March 2021).

31. Li Q, Nie J, Wu J, et al. SARS-CoV-2 501Y.V2 variants lack higher infectivity but do have immune escape. Cell. 2021; 184(9):2362-2371. Available at https://doi.org/10.1016/i.cell.2021.02.042 (accessed 1 May 2021).

458 32. Montagutelli X, Prot M, Levillayer L, et al. The B1.351 and P.1 variants extend SARS-CoV-2 host range to mice. bioRxiv. 2021; 2021.03.18.436013. Available at https://doi.org/10.1101/2021.03.18.436013 (accessed 1 May 2021).

461 33. Faria NR, Claro IM, Candido D, et al. Genomic characterisation of an emergent SARS-CoV-2 lineage in Manaus: preliminary findings. Virological. 2021. Available at 
34. Lucaci AG, Zehr JD, Shank SD, et al. RASCL: Rapid assessment of SARS-COV-2 clades enabled through molecular sequence analysis and its application to B.1.617.1 and B.1.617.2. Virological. 2021. Available at https://virological.org/t/rascl-rapid-assessment-of-sars-cov-2-clades-enabledthrough-molecular-sequence-analysis-and-its-application-to-b-1-617-1-and-b-1-617-2/709 (accessed 29 May 2021).

35. Wan Y, Shang J, Graham R, et al. Receptor recognition by novel coronavirus from Wuhan: an analysis based on decade-long structural studies of SARS coronavirus. Journal of Virology. 2020;

474 36. Damas J, Hughes GM, Keough C, et al. Broad host range of SARS-CoV-2 predicted by comparative 94(7):e00127-20. Available at https://doi.org/10.1128/JVI.00127-20 (accessed on 1 December

37. Flocco MM, Mowbray SL. Planar stacking interactions of arginine and aromatic side-chains in proteins. Journal of Molecular Biology. 1994; 235(2):709-717. Available at https://doi.org/10.1006/imbi.1994.1022 (accessed 1 December 2020).

38. Starr TN, Greaney AJ, Hilton SK, et al. Deep mutational scanning of SARS-CoV-2 receptor binding domain reveals constraints on folding and ACE2 binding. Cell. 2020; 182(5):1295-1310.e20. Available at https://doi.org/10.1016/j.cell.2020.08.012 (accessed 1 December 2020).

39. Piplani S, Singh PK, Winkler DA, Petrovsky, N. In silico comparison of SARS-CoV-2 spike protein- 
41. Bauer DC, Metke-Jimenez A, Maurer-Stroh S, et al. Interoperable medical data: the missing link for understanding COVID-19. Transboundary \& Emerging Diseases. 2021; 68(4):1753-1760. Available at https://onlinelibrary.wiley.com/doi/10.1111/tbed.13892 (accessed 1 July 2021).

42. Priyadarshini, S. Massive coronavirus sequencing efforts urgently need patient data. Nature India (special issue \#13 on COVID-19 crisis). 2020; 10.1038/nindia.2020.75:11-13. Available at https://www.natureasia.com/en/nindia/pdf/special-issues/13/Nature-India-COVID-19-Crisis.pdf and https://go.nature.com/2y7kUlw (accessed 1 December 2020).

43. NSW Government. Help for regional communities impacted by the mouse plague. 2021. Available at https://www.nsw.gov.au/initiative/mouse-control-support-program (accessed 24 May 2021).

44. Zhao X, Chen D, Szabla R, et al. Broad and differential animal angiotensin-converting enzyme 2 receptor usage by SARS-CoV-2. Journal of Virology. 2020; 94(18):e00940-20. Available at https://doi.org/10.1128/JVI.00940-20 (accessed 1 December 2020).

45. Phillips JC, Braun R, Wang W, et al. Scalable molecular dynamics with NAMD. Journal of Computational Chemistry. 2005; 26:1781-1802. Available at https://doi.org/10.1002/icc.20289 (accessed 1 December 2020).

46. Huang J, Rauscher S, Nawrocki G, et al. CHARMM36m: an improved force field for folded and intrinsically disordered proteins. Nature Methods. 2017; 14:71-73. Available at: https://doi:10.1038/nmeth.4067 (accessed 1 December 2020). 
bioRxiv preprint doi: https://doi.org/10.1101/2021.08.04 455042; this version posted December 8, 2021. The copyright holder for this preprint (which was not certified by peer review) is the author/funder, who has granted bioRxiv a license to display the preprint in perpetuity. It is made available under aCC-BY-NC-ND 4.0 International license.

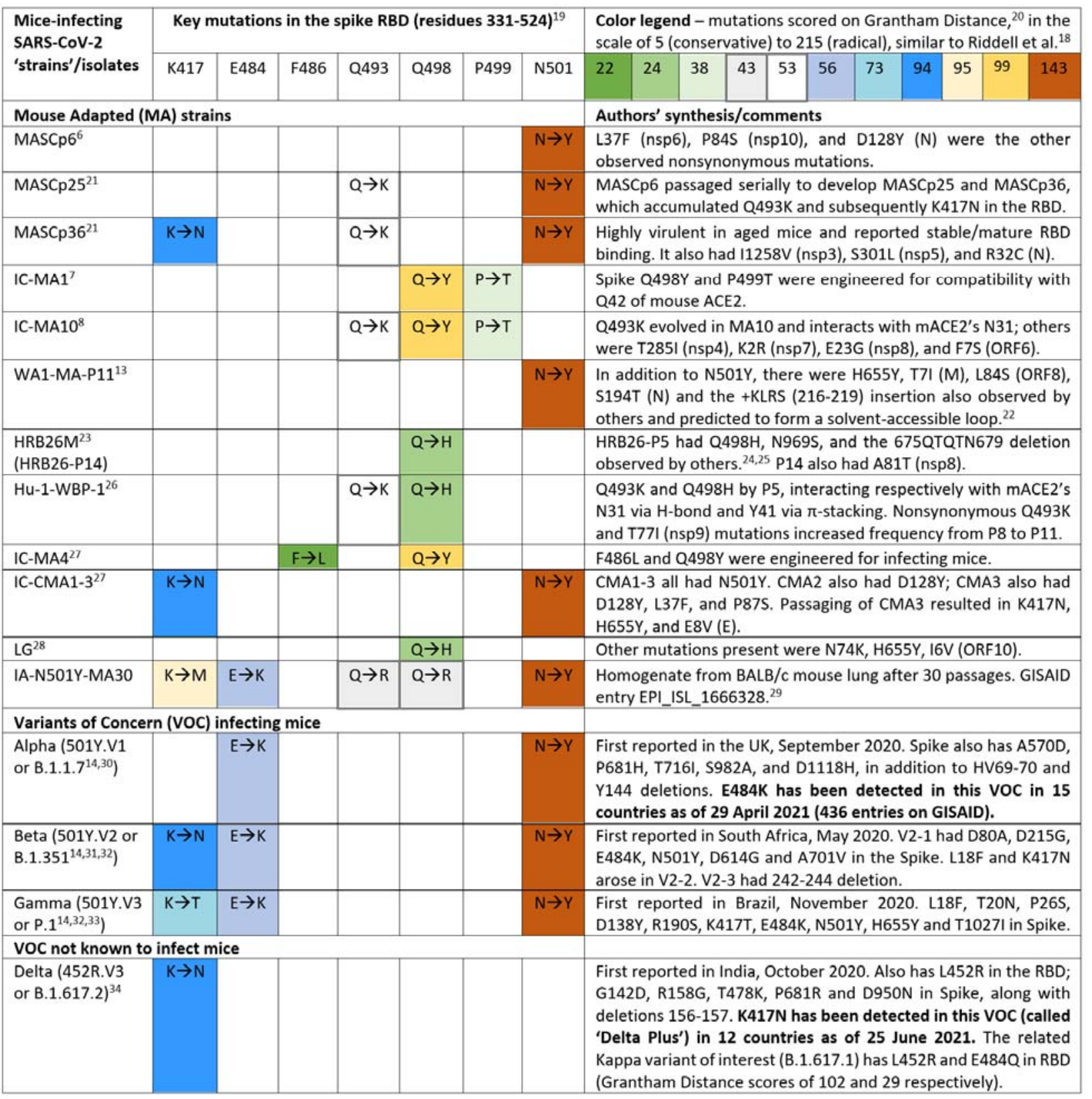

Table 1 Key mutations in 14 'mouse adapted strains' and 3 'variants of concern' of SARS-CoV-2 known to infect mice, with further characterization as outlined in Table 2.

\section{[Provided as a separate word file]}

Table 2. Description of the characteristics of the mouse adapted strains generated from the in vivo studies discussed in Table 1. *Strain = Mouse adapted strain name; Dose = Dose of SARS-CoV-2 mouse adapted strain provided to the mice stated in plaque forming units (PFU) or the tissue culture infective Dose (TCID50); Shedding \& Transmission = Any evidence of viral shedding and viral transmission observed in the mice. 


\begin{tabular}{|c|c|c|}
\hline & $\begin{array}{l}\text { SARS-CoV-2 } \\
\text { RBD mouse adaptations }\end{array}$ & human/mouse ACE2 interface residues within $5 \AA$ of mutation \\
\hline \multirow{3}{*}{$\begin{array}{l}\text { Region } \\
1\end{array}$} & N501 Y & 38D/D, 41Y/Y, 353K/H, 355D/D, 357R/R \\
\hline & P499 T & $41 Y / Y, 45 L / L, 329 E / A, 330 N / N$ \\
\hline & Q498 H/R/Y & $41 Y / Y, 42 Q / Q, 45 L / L, 49 N / N, 61 N / K, 357 R / R$ \\
\hline \multirow{2}{*}{$\begin{array}{l}\text { Region } \\
2\end{array}$} & Q493 K/R & 34H/Q, 35E/E, 38D/D, 42Q/Q \\
\hline & K417 M/N/T & $30 \mathrm{D} / \mathrm{N}, 34 \mathrm{H} / \mathrm{Q}$ \\
\hline \multirow{2}{*}{$\begin{array}{l}\text { Region } \\
3\end{array}$} & E484 K/Q & $35 E / E, 75 E / E$ \\
\hline & F486 L & 75E/E, 76Q/Q, $78 \mathrm{~T} / \mathrm{K}, 79$ L/T, $82 \mathrm{M} / \mathrm{S}$ \\
\hline
\end{tabular}

529 Table 3. SARS-CoV-2 spike RBD mutations associated with mouse adaptation and their close contact 530 residues in respective ACE2 proteins. Positions with dissimilar human/mouse residues are highlighted in 531 yellow. 


\begin{tabular}{|l|l|l|l|l|l|l|l|}
\hline & \multicolumn{3}{|l|}{ Essential position in RBD } & \multicolumn{4}{l|}{ Enhancing position(s) in RBD for mouse adaptation } \\
\hline Analysis & Q498 & N501 & K417 & E484 & F486 & Q493 & P499 \\
\hline In silico [1] & H Y F W & Y H F W [5] & N Q [8] & K Q & L & K L R & T \\
& [5] & & & & & & \\
\hline In vitro [2] & H Y F W & F Y V W T & - & R K T Q & - & M A Y F K L & - \\
\hline In vivo [3] & H R Y & Y & M N T & K Q & L & K R & T \\
\hline In situ [4] & R H [6] & Y T H F [7] & N T M [9] & K Q R T [10] & L [11] & K L R [12] & T [13] \\
\hline 1. Predice by & & & & & & & \\
\hline
\end{tabular}

1. Predicted by our modelling. For E484, F486, Q493, and P499 our work only reconfirmed in vivo observations.

2. Arranged in descending order of affinity binding from in vitro experiments reported by Starr et al. ${ }^{38}$

3. Observed in vivo with studies summarized in Table 1 (alphabetical order). Note L486 and P499 were engineered.

4. Observed on GISAID as of 21 July 2021 after removing noise from low sample countries. Only key trends are presented.

5. These aromatic residues are predicted to have similar interactions with Y41 of ACE2.

6. F W Y not observed yet. Since early 2021, R observed in several countries, and $\mathrm{H}$ in Slovenia and USA (albeit the latter are of low quality and/or low coverage).

7. V W not observed yet. H observed in several countries (mid 2020), T in Spain's Canary Islands (early 2021), and F in Colombia, Germany, Mexico and Sweden (mid 2021). Y observed in several countries (Figure S1).

8. Modelled mutations predict favorable amide hydrogen bonding. $\mathrm{N}$ and $\mathrm{Q}$ mutations each require a single nucleotide change but there are 2 ways to get from $\mathrm{N}$ to $\mathrm{K}$.

9. $\mathrm{Q}$ observed in the USA (August 2021). N (mutation G22813T) and T observed several countries (Figures S2a and S2b). Interestingly, N (alternative mutation G22813C) has a sporadic presence in UK and USA (since late 2020).

10. K observed in several countries (Figure S2c). Sporadic presence of $Q$ (several countries from March 2020 to August 2021), R (Angola, Brazil, South Africa, South Korea, UK, USA, since late 2020), and T (USA in mid 2021) observed.

11. Sporadic presence of different synonymous mutations ( $T 23018 \mathrm{C}$ in several countries; T23020G in 
USA and one instance in Turkey) observed since late 2020.

12. A, F, M, V, Y not observed yet. Sporadic presence observed for K (Italy since early 2020), L (Trinidad and Tobago in mid 2021), and R (several countries mid 2020).

13. Sporadic presence since late 2020 in Bulgaria, Canada, Netherlands and USA.

536 Table 4. Comparison of in silico, in vitro, in vivo and in situ observations of key mutations in Table 1. 


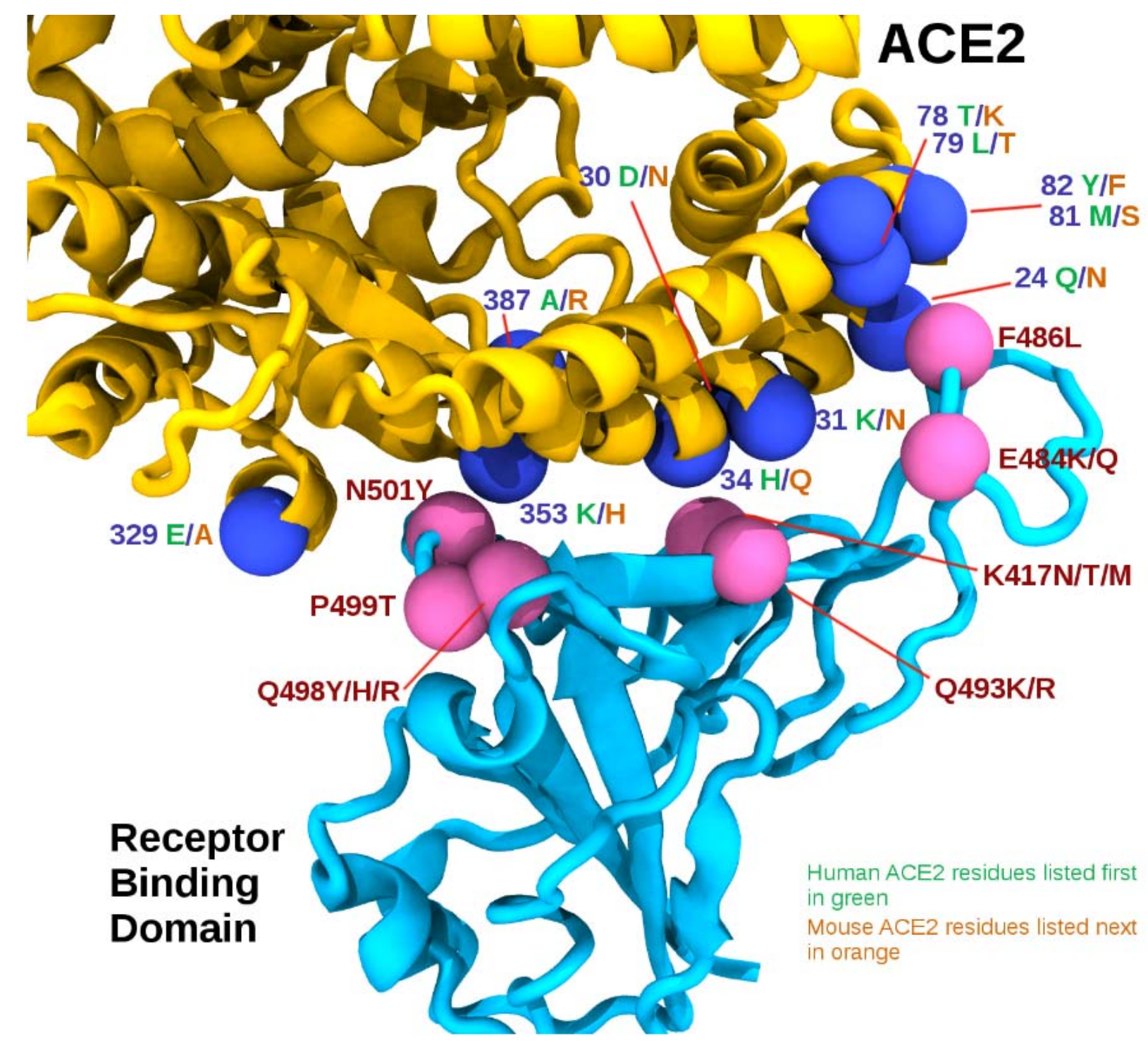

539 Figure 1. The RBD/ACE2 interface. The receptor binding domain (RBD) is shown in cyan, while the ACE2

540 is shown in yellow. Pink spheres indicate relative positions of mouse adapting mutations on the RBD,

541 while the blue spheres represent interface residues that differ between human and mouse ACE2

542 sequences, shown in green and orange respectively. 
bioRxiv preprint doi: https://doi org/10.1101/2021.08.04 455042. this version posted December 8,2021 . The copyright holder for this preprint (which was not certified by peer review) is the author/funder, who has granted bioRxiv a license to display the preprint in perpetuity. It is made available under aCC-BY-NC-ND 4.0 International license.

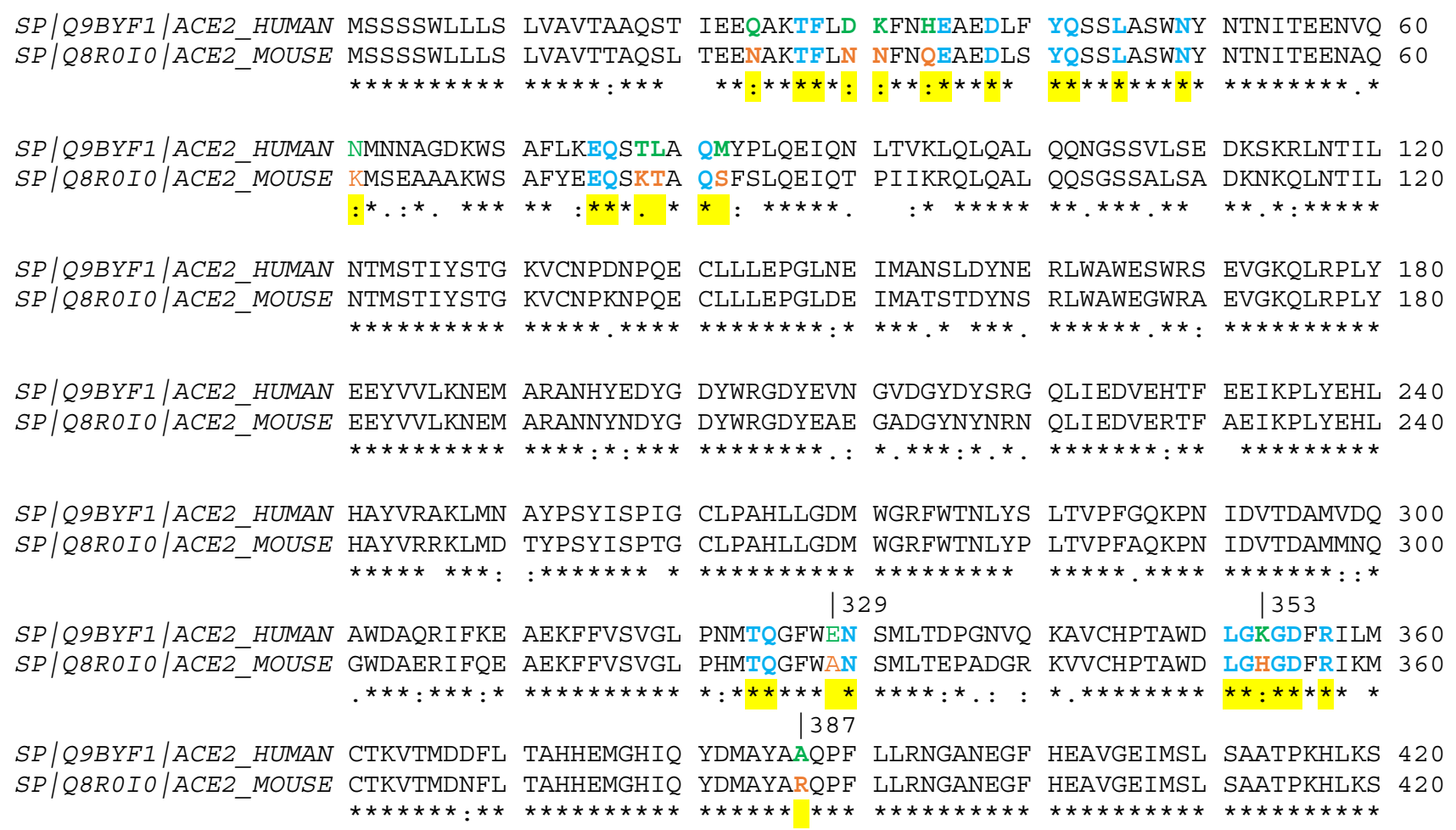

546 Figure 2 Sequence alignment of human and mouse ACE2 highlighting contact points with the SARS-

547 CoV-2 spike receptor binding domain in yellow. Differences between contact points are highlighted in

548 green (human) and orange (mouse), while common contact residues are highlighted in cyan. 


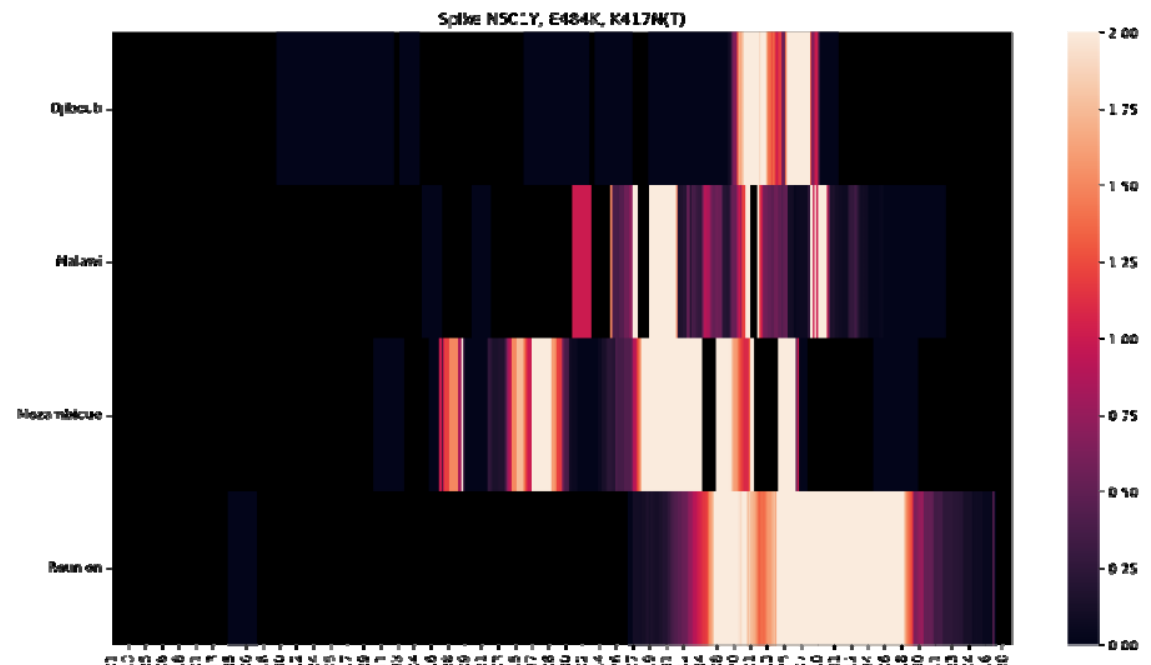

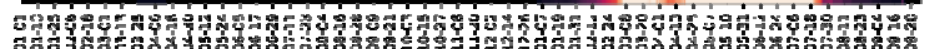

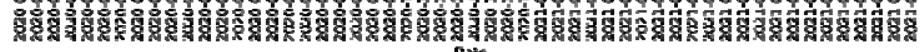

551 a)

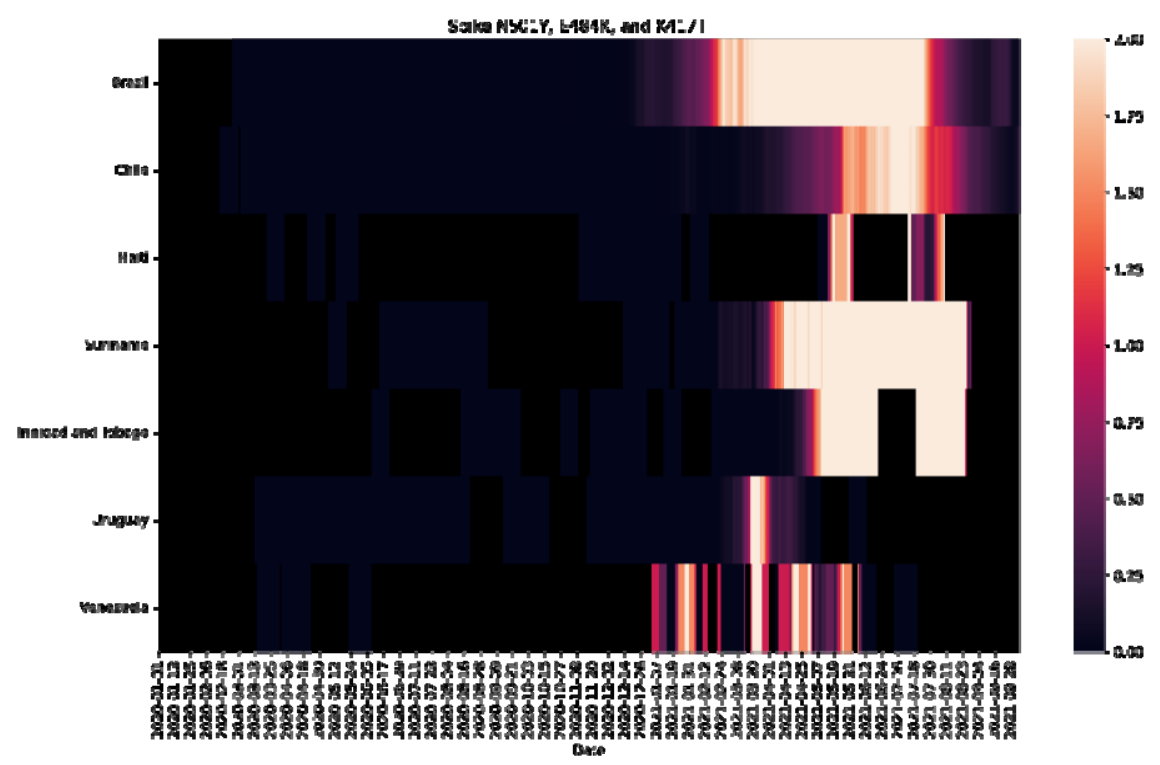

553 b)

554 Figure 3. Significant occurrences on GISAID of a) N501Y, E484K and K417N (nucleotide change G22813T)

555 triple mutations and b) N501Y, E484K and K417T triple mutations, at the virus Receptor Binding Domain

556 (RBD) since the start of the COVID-19 pandemic (31 December 2019). These countries are encouraged to

557 perform targeted field surveillance. 
bioRxiv preprint doi: https://doi.org/10.1101/2021.08.04 455042; this version posted December 8, 2021. The copyright holder for this preprint (which was not certified by peer review) is the author/funder, who has granted bioRxiv a license to display the preprint in perpetuity. It is made available under aCC-BY-NC-ND 4.0 International license.

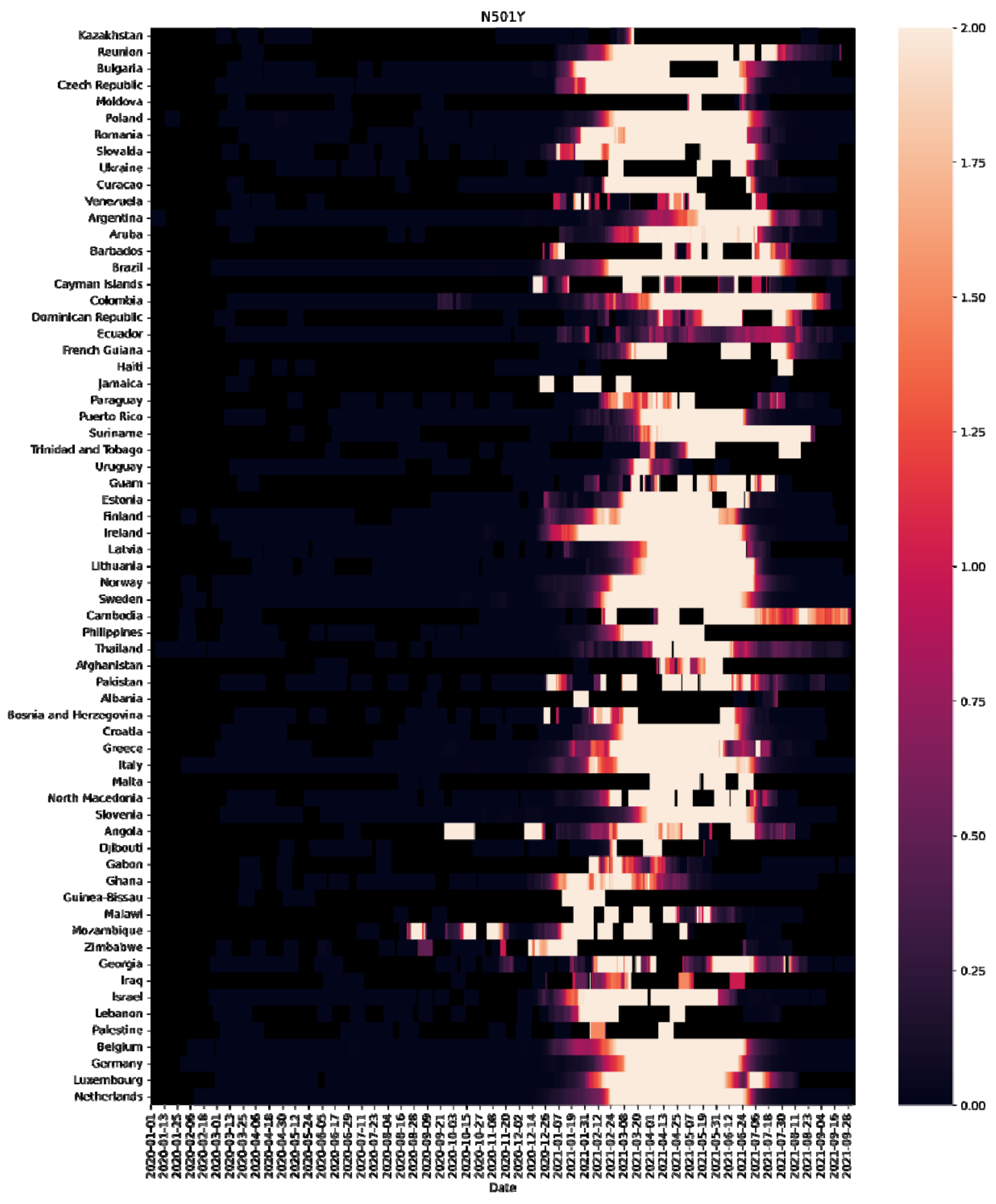

559 Figure S1. Significant occurrences on GISAID of the essential N501Y mutation (nucleotide change

560 A23063T), at the virus Receptor Binding Domain (RBD) since the start of the COVID-19 pandemic (31

561 December 2019). 
bioRxiv preprint doi: https://doi.org/10.1101/2021.08.04.455042; this version posted December 8, 2021. The copyright holder for this preprint (which was not certified by peer review) is the author/funder, who has granted bioRxiv a license to display the preprint in perpetuity. It is made available under aCC-BY-NC-ND 4.0 International license.

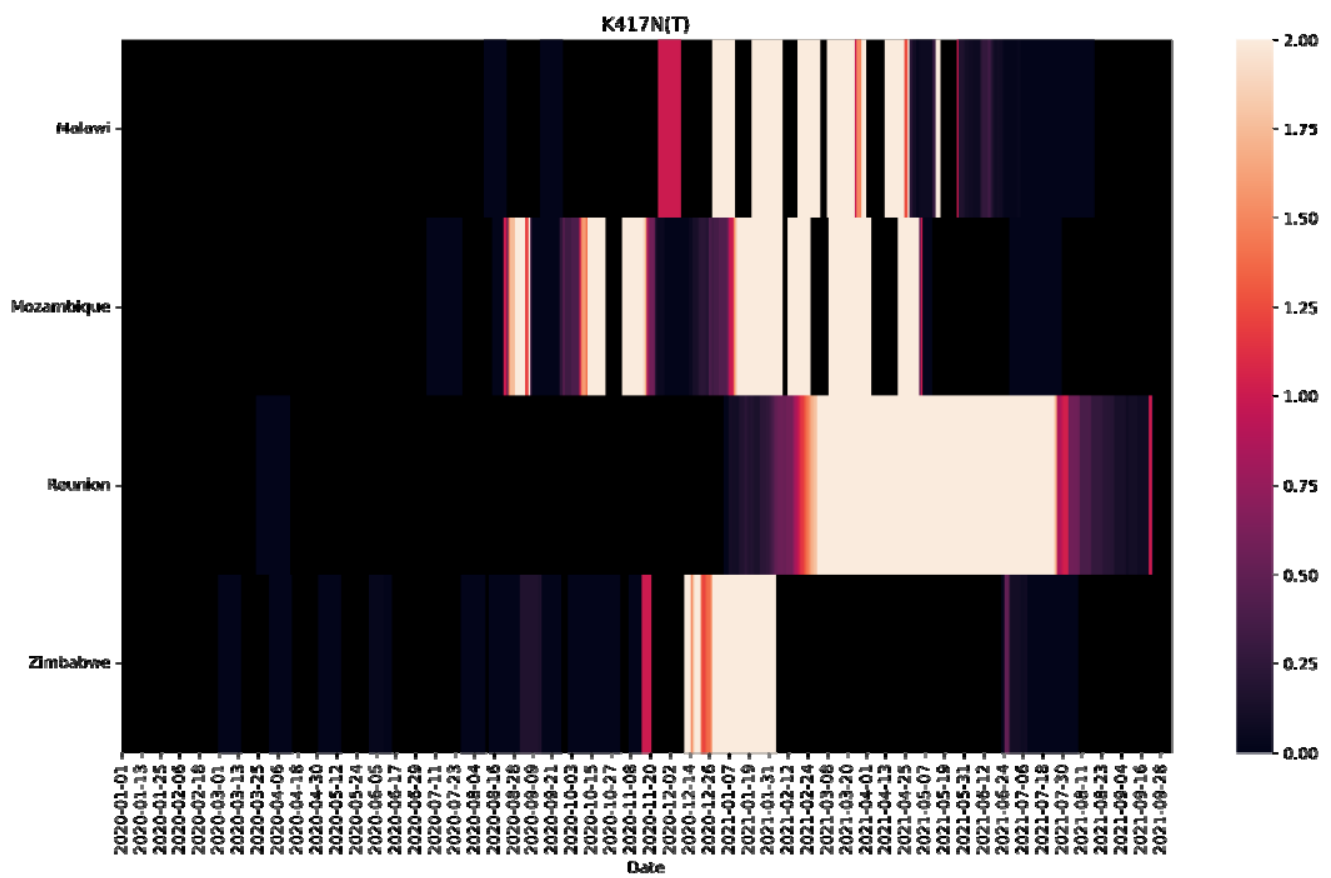

564 a)

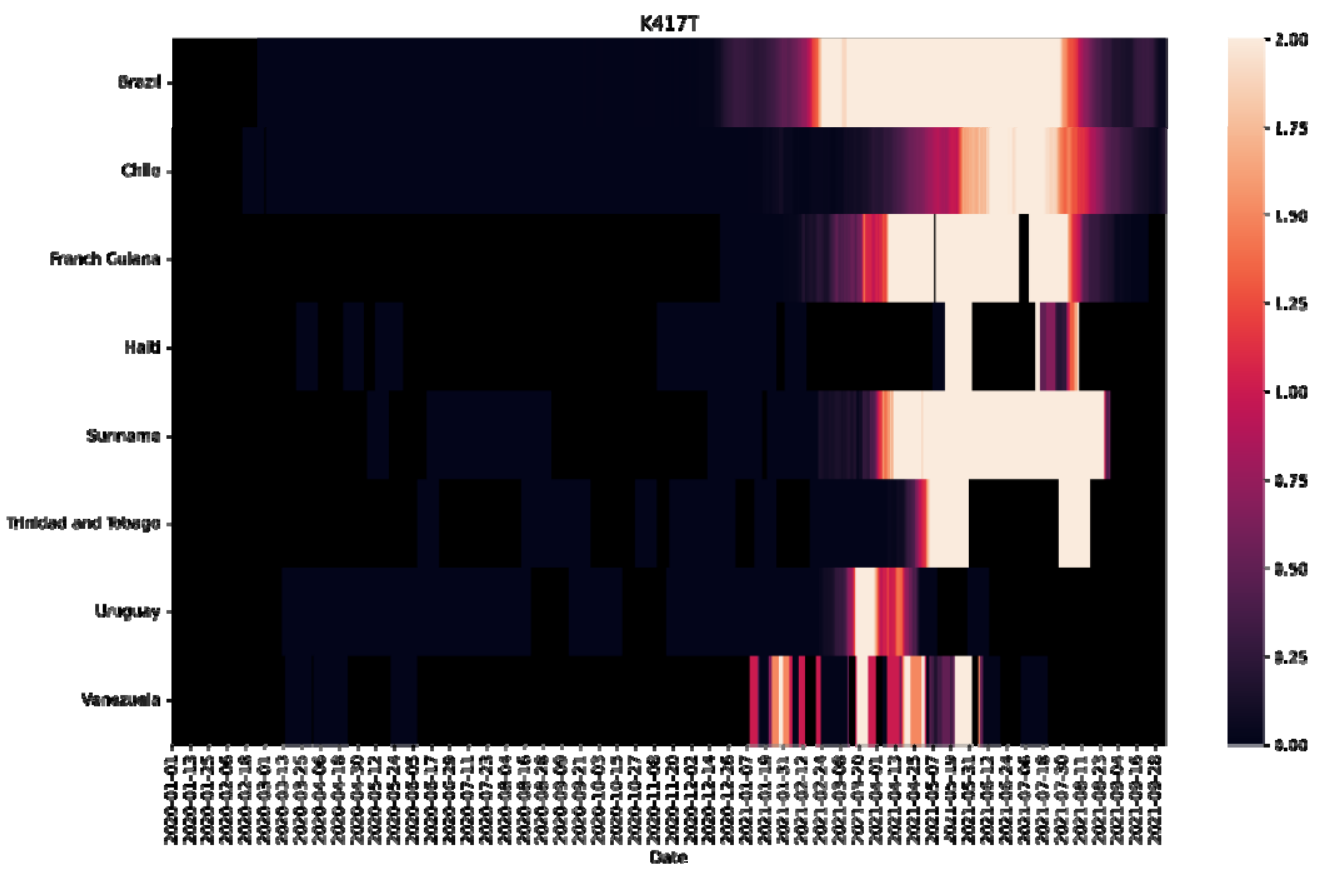

\section{6 b)}




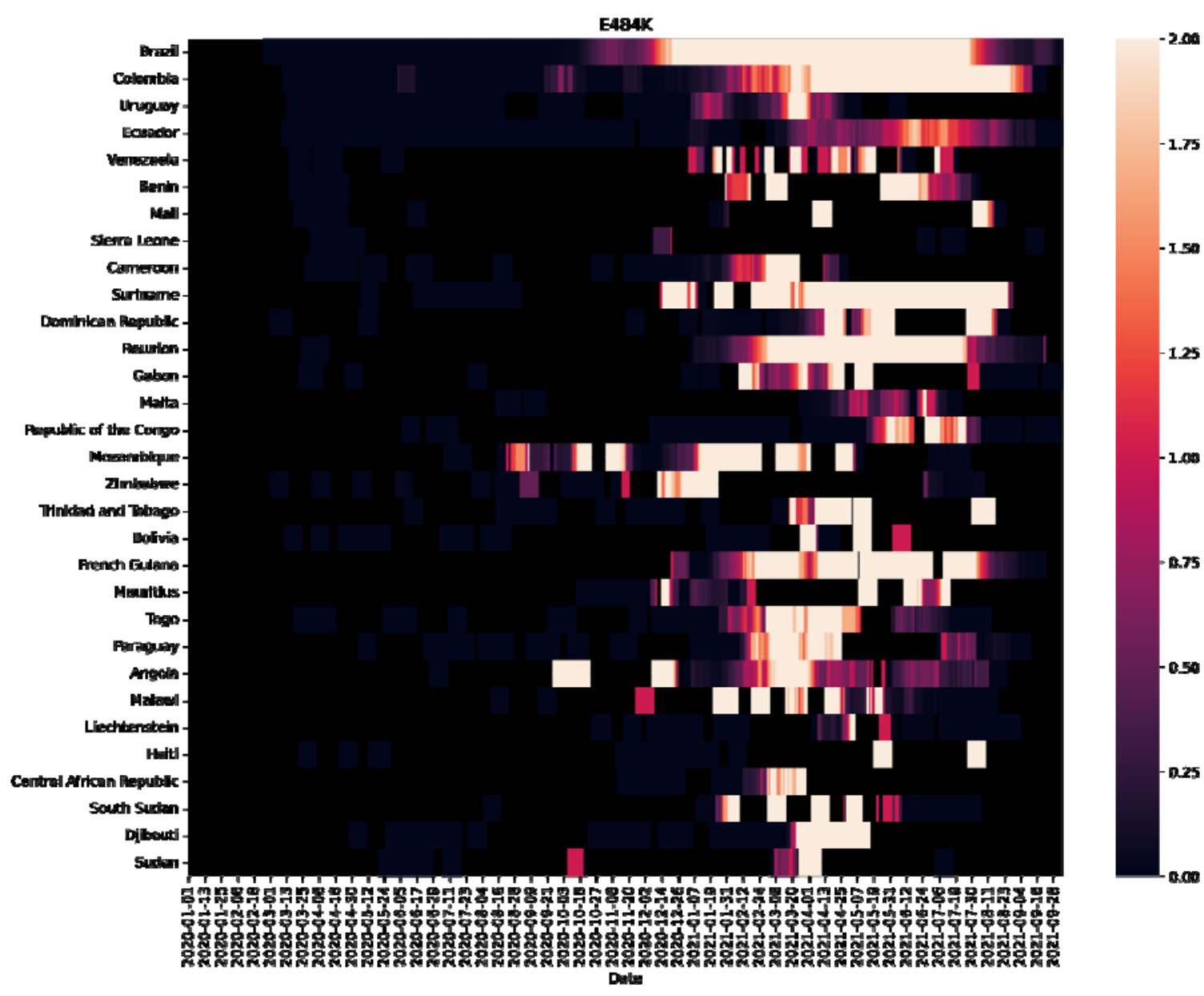

569 c)

571 Figure S2. Significant occurrences on GISAID of the enhancing mutations a) K417N (nucleotide change

572 G22813T), b) K417T (nucleotide change A22812C), c) E484K (nucleotide change G23012A), at the virus

573 Receptor Binding Domain (RBD), since the start of the COVID-19 pandemic (31 December 2019). 


\section{Species and age of mice: Aged (9 months old) and} Species and age of mice: Aged (9 months
young ( 6 weeks old) BALB/c mice studied.

\section{Strain name: MASCp $6^{6}$}

Dose: Intranasal inoculation with $1.6 \times 10^{4} \mathrm{PFU}$

Shedding: Viral shedding observed in the feces at day 3,5 and 7 after inoculation.

Transmission: No information

Species and age of mice: Young (8-week-old) and aged ( 9 months old) male and female BALB/c mice and C57BL/6 mice studied.

\section{Strain name: MASCp36 ${ }^{21}$}

Dose: Intranasal inoculation with varying doses including: 1.2 PFU, 12 PFU, 120 PFU, 1200 PFU, 12000 PFU

\section{Shedding: No information}

Transmission: No information Species and age of mice: Young adult (12 weeks old) and aged (One year old) BALB/c mice studied. ${ }^{7}$

\section{Strain name: IC-MA1 ${ }^{7}$}

Dose: Intranasal inoculation with $10^{5} \mathrm{PFU}$

Shedding: No information

Transmission: No information

\section{Species and age of mice: Young (10-week-old) and} aged ( 1 year old) BALB/c mice studied. ${ }^{8}$

\section{Strain name: IC-MA $10^{8}$}

Dose: Doses ranging from $10^{2}, 10^{3}, 10^{4}$ or $10^{5} \mathrm{PFU}$ were tested. However, $10^{4}$ PFU and $10^{3}$ PFU were determined as the optimal doses for analysis in young and aged mice respectively.

Shedding: Both old and young mice exhibited viral titers in the nasal cavity over the first 3 days of infection.

\section{Transmission: No information}

Species and age of mice: $\mathrm{C} 57 \mathrm{~B} \mid 6, \mathrm{BALB} / \mathrm{C}$ and
129S1/SVMJ mice were studied

Young (6-8-weeks) or aged (52 weeks old) C57BI6 mice were used to study the effect of obesity, obesityassociated diabetes, and advanced age on clinical
High amounts of viral RNAs in the lungs and tracheas were detected at 3,5 and 7 days after inoculation in all aged mice, with peak viral RNA loads of $\sim 10^{10} \mathrm{copies} / \mathrm{g}$ at 3 days after inoculation. Viral RNAs were also detected in heart, liver, spleen, and brain.

Similar tissue distribution of SARS-CoV-2 RNA was also seen in the MASCp6 infected young mice.

qRT-PCR results of aged BALB/c and C57BL/6 mice challenged with 12,000 $\square \mathrm{PFU}$ of MASCp36 showed that high levels of SARS-CoV-2 subgenomic RNAs were persistent in the lung and tracheas till $4 \mathrm{dpi}$. A similar tissue distribution of SARSCoV-2 was observed in the young BALB/C and C57BL/6 mice.

High-titre virus replication was also observed in lung tissue at $2 \mathrm{dpi}$. This cleared by $4 \mathrm{dpi}$ in the young adult mice but continued to persist at $4 \mathrm{dpi}$ in the aged mice, suggesting that there was increased viral replication in the aged mice.

MA1 was also observed in the upper airway and viral antigen was present in nasal turbinate epithelium at $2 \mathrm{dpi}$ in young adult mice; viral antigen was found in conducting airway epithelium, interstitium and nasal epithelium in aged mice at 4 dpi.

Virus replication in the lungs of young mice peaked 1-2 dpi and was absent in most surviving mice by $7 \mathrm{dpi}$. Viral replication in the upper respiratory tract of young mice remained high on 1-3 dpi but was undetectable in most mice by $5 \mathrm{dpi}$.

A similar trend was observed in the aged mice, but the viral replication was detectable for a much longer period.

Detectable virus titres in lungs and nasal turbinates of 129S1/SVMJ mice and lungs of male and female C57BI6 and BALB/c mice, obtained at different days post infection
Clinical: No visible clinical symptoms or body weight loss were observed in both the young and aged mice. No fatalities recorded.

Histopathological: Led to interstitial pneumonia and inflammatory responses in both young and aged mice, however the lung damage in the aged mice was more severe.

Clinical: All the aged BALB/C and C57BL/6 mice challenged with high doses (1200 or 12,000ロPFU) of MASCp36 developed typical respiratory symptoms and exhibited features like ruffled fur. hunched back, and reduced activity.

Young BALB/c and C57BL/6 mice were resistant to MASCp36 challenge, and only one animal that received $12,000 \square \mathrm{PFU}$ challenge died during the observation period.

Histopathological: Necrotizing pneumonia and extensive diffus alveolar damages observed on $4 \square \mathrm{dpi}$ in aged mice. Milder pathology observed in the young mice.

Clinical: No overt clinical signs such as weight loss observed in young adult mice but a significant decrease in body weight was observed in aged mice.

Whole-body plethysmography on mice indicated impaired lung function at $2 \mathrm{dpi}$, with the extent of impairment significantly worse in the aged mice.

Histopathological: Interstitial congestion, epithelial damage, inflammatory infiltrate and peribronchial lymphocytic inflammation surrounding airways at $2 \mathrm{dpi}$. Histopathological effects seen in the aged mice are more severe than those observed in young adult mice.

Clinical: Young mice rapidly lost weight and reached maximum weight loss at day 4 post inoculation. On day 5 , the collective mortality rate was approximately $15 \%$. Significant weight loss was also observed in aged mice.

Mortality rates of $20 \%$ and $60 \%$ were recorded for infection with $10^{4}$ and $10^{5} \mathrm{PFU}$, respectively in the young mice.

Histopathological: At the time of necropsy, acute stage lung damage was noted in the young mice.

Analysis at 2, 4, and 7 dpi revealed early multifocal damage to conducting airway epithelia and hallmarks of diffuse alveolar damage (DAD) was observed. The aged mice had severe DAD and higher acute lung injury scores. Overall, a dose- and agerelated increase in pathogenesis was observed.

Clinical: Weight loss detected in the 129S1/SVMJ mice but not in the C57BI6 and BALB/c mice.

Effect of increased age, comorbidities: Both obesity/diabetes and advanced age in mice result in higher morbidity during SARS- 
Strain name: WA1-MA-P $11^{13}$

Dose: Intranasal inoculation with $2.5010^{4}$ PFU

Shedding: No information

Transmission: No information

Species and age of mice: Groups of young (4-6-weekold) female BALB/c mice, young (4-6-week-old female) C57BL/6J mice and aging (8-9-month-old) male BALB/C mice studied.

\section{Strain name: HRB26M ${ }^{23}$}

Dose: Intranasal inoculation with $10^{4.4} \mathrm{PFU}$ in a volume of $50 \mu \mathrm{L}$.

\section{Shedding: No information}

Transmission: No direct evidence of transmission provided. However, the presence of efficient viral replication in the upper and lower respiratory tracts indicates that transmission could be possible.

Species and age of mice: Young (4-6-week-old) $\mathrm{BALB} / \mathrm{c}$ mice studied.[Female 12-month-old BALB/C mice only used for the initial generation of the mouse adapted strain, not for any analysis of the mouse adapted strain]. ${ }^{26}$

Strain name: $\mathrm{Hu}-1-\mathrm{WBP}-1^{26}$

Dose: Intranasally infected with 10 -fold serial dilutions of the WBP-1 virus (i.e., $10^{2}$ to $10^{5} \mathrm{PFU}$ ).

Subsequently, detailed analysis on mice was done by infecting mice with $50 \mu \mathrm{L}$ of $2 \mathrm{LD}_{50}$ [The $\mathrm{LD}_{50}$ of WBP-1 is $10^{3.8} \mathrm{PFU}$.

Shedding: No information

Transmission: No information Species and age of mice: 10 -week-old female BALB/C mice).

\section{Strain name: IC-MA4}

Dose: Mice infected with $10^{5} \mathrm{PFU}$

Shedding: No information

Transmission: No information

Species and age of mice: 10-week-old female BALB/C mice. $^{27}$

Strain names: IC-CMA $1^{27}$, IC-CMA2 $2^{27}$, IC-CMA3 ${ }^{27}$ Dose: Mice intranasally inoculated with $10^{5} \mathrm{PFU}$

\section{Strain names:}

In young BALB/c mice, viral RNA was detected in the nasal turbinates on day 3, 5 , and 7 p.i. (post infection) and the infectious virus was detected on day 3 and 5 p.i. The viral RNA was also detected in the heart, liver, kidney, and spleen on day 3 p.i.

In C57BL/6J mice, high viral loads in the nasal turbinates and lungs on days 3, 5 and 7 p.i.

Similar observations made in the aging mice. However, when compared to the young mice, the average PFU titres in the aging mice were 3 times higher and 10 times higher in the nasal turbinates and lungs respectively.

In mice given the $50 \mu \mathrm{L}$ of $2 \mathrm{LD}_{50}$ : High viral loads were observed in the trachea and lung of infected mice, with lung tissues having the highest number of viral RNA copies. Trace amounts of viral nucleic acid were detectable in turbinate, heart, and spleen.

Evidence of robust viral replication in mouse lungs.

All three mutants (CMA1, CMA2, CMA3) produced approximately, $10^{5}$ PFU per lobe at day 2 post infection. However, no virus was detected at day 4 , suggesting rapid clearance by the host.

In mice given IC-CMA3p20, robust viral replication was observed. There was a significantly greater viral load in the lung compared to IC-CMA3p20 at $2 \mathrm{dpi}$.
Clinical: Similar to the young mice, the aging adult mice showed transient weight loss on days 2 and 3 p.i. and recovered thereafter.

Histopathological: Mild pathological changes were observed in the respiratory tract of young BALB/c mice infected.

Moderate-to-severe pathological changes in the lungs after infection in the aging mice.

Clinical: In the groups of mice infected with the 10 -fold serial

dilutions: All groups of infected mice began to lose weight at 2 dp and the weight loss was dose dependent. Bristled fur and depressed spirits in mice was also observed.

The group of mice receiving a dose of $10^{5}$ PFU WBP-1 virus all died $(5 / 5)$ by 5 dpi. Two out of five mice survived in the group administered $10^{4}$ PFU virus.

In the mice given the $50 \mu \mathrm{L}$ of $2 \mathrm{LD}_{50}$ : Clinical signs of infection were observed in infected mice at $2 \mathrm{dpi}$

Histopathological: In mice given the $50 \mu \mathrm{L}$ of $2 \mathrm{LD}_{50}$, Acute stag lung damage was noted. Moderate interstitial pneumonia with thickened alveolar septa occurred at $3 \mathrm{dpi}$, which progressed to severe interstitial pneumonia on $5 \mathrm{dpi}$. Inflammatory cell infiltratiol was observed in the lung tissue.

\section{Clinical: No information}

Histopathological: No information

Clinical: None of the three mutants (CMA1, CMA2, CMA3)

induced major disease, although both CMA2 and CMA3 caused more weight loss than CMA1.

Mice infected with IC-CMA3p20 had a 10\% weight loss by day 3 and showed signs of disease including ruffled fur and hunched posture. 


\section{[IC-CMA3 after passage 20] \\ Dose: $10^{5}$ PFU was the main dose administered, but} doses $10^{4}$ and $10^{6} \mathrm{PFU}$ also administered to some mice.

Species and age of mice: Young (2 months old) and aged (12-month-old) BALB/c mice. ${ }^{28}$

Effective viral replication in the lungs and trachea of the young and aged mice, although there was more viral replication in the aged mice.
Histopathological: Mice infected with CMA3p20 had significant lung infiltration and inflammation characterized by peribronchiolar, perivascular cuffing, and perivasculitis by day 2 post infection. At 4 dpi, collapsed airways and interstitial pneumonia was observed in the mice.

Clinical: Weight of the aged mice was slightly decreased, but there were no evident changes in the weight of young mice.

Histopathological: Lung lesions of the aged BALB/c mice were obvious, but those of young mice were not, with only slight lymphocyte exudation.

Dose: $1 \square \times \square 10^{5} \mathrm{TCID}_{50}$ in a volume of $50 \square \mu \mathrm{l}$.

Shedding: No transmission

Transmission: No information

Table 2: Description of the characteristics of the 'mouse adapted strains' generated from the in vivo studies discussed in Table 1. ${ }^{*}$ Strain = Mouse adapted strain name, Dose = Dose of SARS-CoV-2 mouse adapted strain provided to the mice stated in plaque forming units (PFU) or the Tissue culture infective dose (TCID50), Shedding \& Transmission = Any evidence of viral shedding and viral transmission observed in the mice 